\title{
Phytoprotection
}

\section{Index des sujets, volume 82 Subject Index, Volume 82}

Volume 82, numéro 3, 2001

URI : https://id.erudit.org/iderudit/706225ar

DOI : https://doi.org/10.7202/706225ar

Aller au sommaire du numéro

Éditeur(s)

Société de protection des plantes du Québec (SPPQ)l

ISSN

0031-9511 (imprimé)

1710-1603 (numérique)

Découvrir la revue

Citer ce document

(2001). Index des sujets, volume 82. Phytoprotection, 82(3), 143-144.

https://doi.org/10.7202/706225ar d'utilisation que vous pouvez consulter en ligne.

https://apropos.erudit.org/fr/usagers/politique-dutilisation/ 


\section{Index des sujets, volume 82 \\ Subject Index, Volume 82}

\section{A-B}

Acide abscissique / abscissic acid

Actinomycètes / Actinomycetes

Agropyron repens

Agrostide

Agrotis ipsilon

Alberta

Alfalfa

Annual changes

Aphodius sp.

Apple orchard

Application localisée d'herbicides

Ataenius spretulus

Attract and kill technique

Azoxystrobin / azoxystrobin

Bacillus thuringiensis

Barley

Bentgrass

Benzimidazole

Bio-encapsulation / bio-encapsulation

Biological control

Bipolaris sorokiniana

Blé

Botrytis

cinerea

squamosa

Brassica

juncea

rapa

$\beta$-tubulin

\section{C-E}

Ceratocystis resinifera

Champignons

Changements annuels

Choristoneura rosaceana

Coated microbial pesticide

Contrôle des mauvaises herbes

Cronartium comptoniae

Détection / detection

Diabrotica virgifera virgifera

Distribution des chrysalides

Écosystème urbain

Endophytes

Endophytic ryegrass
Entomopathogenic nematode

49

Épidémie

57

Extension

35

\section{F-L}

Fludioxonil / fludioxonil

Formulation / formulation

73

Fragaria $\mathrm{x}$ ananassa

129

Fumigation / fumigation

13

Fungicide resistance

103,134

Fusarium sp.

131

Gaeumannomyces graminis

var. avenae

125

Gazon

134

Golf lands

133

Helminthosporium solani

Herbicide local spraying

$41,103,128$

Horismenus fraternus

125

65

Imazalil/ imazalil

41

Insecticide resistance $\quad 113$

Integrated control $\quad 124$

Inventaire

49

Iprodione / iprodione

41

57

113

13

133

85,134

124

130

\section{M-O}

Mancozeb / mancozeb

Manitoba

Methyl jasmonate

Mycoflora

Nématode / nematode 
P-R

Parasitisme / parasitism

Peridermium harknessii

Pesticide microbien enrobé

PGPR

Pholetesor ornigis

Phyllonorycter blancardella

Phytophthora

megasperma f. sp. medicaginis

fragariae var. rubi

Picea abies

Piégeage

Pinus banksiana

Pissodes strobi

Plant-parasitic nematode

Pnigalio flavipes

Polymerase chain réaction

Pomme de terre / potato

Pratylenchus penetrans

Puccinia recondite

Pupal distribution

Pythium ultimum

Réaction de polymérisation

en chaîne

Résistance aux fongicides

Résistance aux insecticides

Rhagoletis pomonella

R6 growth stage

R8 growth stage

\section{$\mathbf{S}$}

Saskatchewan

Sclerotinia minor

Secale cereale

Septoria musiva

Silver scurf

Soja

Soja, gousses

Soja, graines

Sorghum

bicolor

vulgare

Soybean

Soybean pods

Soybean seeds

Stade de croissance R6

Stade de croissance R8

Streptomyces

scabies

$s p$.

Streptomycètes / Streptomycetes
Survey

128,130

Sympiesis dolichogaste marylandensis

sericeicornis

\section{T-W}

Tache argentée

Telone / Telone

Terrains de golf

Thiabendazole / thiabendazole

41,103

Tipula sp.

Trifolium pratense 13

Triticum aestivum 13

49 Turfgrass 134

65 Uncinula necator 123

103 Urban ecosystem 124

113 Verger de pommiers 73

13 Vigna radiata 135

135 Vignoble 49

57 Vineyard 49

123 Vorlex Plus CP / Vorlex Plus CP 13

Weed control 134

103. Western Canada 113

103,134 Wheat 131, 135
113

25

1

1

113

134

1,35

1

1

13

13

1, 35

1

1

1

1 